\title{
The Collective Radioactive Decay of Atomic Nuclei Initiated by an External Mechanical Impact: Science Fiction or a New Class of Physical Processes
}

\author{
M. K. Marakhtanov ${ }^{1} \&$ V. S. Okunev ${ }^{2}$ \\ ${ }^{1}$ Department of Plasma Power Installation at the Bauman Moscow State Technical University, 105005, 2th \\ Baumanskaya street, 5, Moscow, Russia \\ ${ }^{2}$ Department of Physics at the Bauman Moscow State Technical University, 105005, 2th Baumanskaya street, 5, \\ Moscow, Russia \\ Correspondence: Okunev V.S., Marakhtanov M.K., Bauman Moscow State Technical University, 105005, 2th \\ Baumanskaya street, 5, Moscow, Russia. E-mail: mkm@bmstu.ru, okunev_vs@mail.ru
}

Received: February 5, 2017

Accepted: March 9, 2017

Online Published: March 31, 2018

doi:10.5539/jmsr.v7n2p34

URL: https://doi.org/10.5539/jmsr.v7n2p34

\begin{abstract}
Abnormally high pressure or temperature can significantly reduce the lifetime of atomic nuclei including stable. A hypothesis on stable nuclei decays caused by mechanical collision of macro-objects containing these nuclei is put forward. Mechanisms of such decays are presented. At the same time the distance between interacting nuclei can be significantly higher than the range of nuclear interaction (as manifestations of strong interaction). In decays initiated by external impact, the mean lifetime with respect to particular decay can change depending on these factors. The hypothesis is verified experimentally. When a bismuth bullet collides with a steel flat target, we see different types of stable bismuth isotope decay including cluster one, which is not found in nature (if there is no external impacts). This new class of physical processes can be related to the collective radioactive decay of atomic nuclei initiated by an external mechanical impact.
\end{abstract}

Keywords: radioactive decay, cluster decay, half-lifetime, magic nucleus, deformed nucleus, inertial explosion of metals, bismuth

\section{Introduction}

\subsection{Background}

The ancient alchemists dreamed on transforming the not-noble metals into gold. Bolos Democritos was the first who voiced this dream 200 years before B.C. And only in XX century it was implemented. Accelerators of charged particles, nuclear reactors and ionizing radiation play a role of a philosopher's stone (the required reagent). The problem is as follows: is it possible to transform nuclei by hammer, high pressure or temperature impacts? There were no other possibilities for the alchemists.

\subsection{Ways for Selecting Material for Experiments}

Among light nuclei, deuterium and beryllium-9, which are characterized by low binding energy of the last neutron, are the most preferable for alchemical experiments. Whether it is possible to transform much heavier atomic nucleus, for example, a stable bismuth isotope loved by alchemists by means of hammer impact or by mechanical collision of macro-objects containing the studied material?

Why bismuth-9 is of interest for a modern researcher? It is the heaviest stable element. The radioactive halflifetime (alpha decay) of bismuth-209 is $T_{1 / 2}=(1.9 \pm 0.2) \cdot 10^{19}$ years (Audi, Wapstra, \& Thibault, 2003). The bismuth-209 nucleus is magical in terms of the number of neutrons $(\mathrm{N}=126)$ and near-magical in terms of the number of protons $(Z=83)$. The bismuth-209 nucleus being in the ground energy state is characterized by a huge half-integer spin $(9 / 2$ (Bhatki, 1977)) and it is deformed. Bismuth-209 is characterized by an optimal neutron-toproton ratio $(\mathrm{N} / \mathrm{Z})$ in terms of equilibrium of nuclear forces (as manifestations of the strong interaction), electromagnetic forces and weak forces: $\mathrm{N} / \mathrm{Z}=0.98+0.015 \mathrm{~A}^{2 / 3}$ (where $\mathrm{A}$ is the mass number, $\mathrm{A}=\mathrm{N}+\mathrm{Z}$ ). Increased stability should be intrinsic to bismuth-209 nucleus. 


\subsection{Two Ways for Transmuting the Atomic Nuclei by External Impact}

There are two ways for transmuting nuclei, if there are no accelerators, nuclear reactors and ionizing radiation.

The first way is to approach nuclei down to the range of nuclear forces $\left(r_{S} \approx 10 \mathrm{fm}\right.$, in the Yukawa theory $r_{S}=1.4$ $\mathrm{fm}($ Okunev, 2015)). This way is preferable for the light nuclei, which approach is prevented by relatively small Coulomb barrier, and it is implemented in thermonuclear fusion reactors.

Another way is to activate the radioactive decays by minimizing factors stabilizing decays. This way is preferred for intermediate- and heavy-mass nuclei. The electron "cloud" protects the nucleus against external impacts. If the cloud is deformed, completely or partially removed, the protection becomes imperfect and external impacts (pressure, including caused by mechanical impact, temperature, etc.) can significantly reduce the half-lifetime.

\section{Hypothesis}

\subsection{Interaction Conditions}

For atomic nuclei interaction it is necessary to surmount the Coulomb barrier preventing their approaching. Under collision of two bismuth-209 nuclei, the simplest estimate of the Coulomb barrier gives $1106 \mathrm{MeV}$. Under collision of ferrum-56 and bismuth-209 nuclei, the Coulomb barrier is $434 \mathrm{MeV}$. It is difficult to surmount such barrier by mechanical collision of macro-objects. If the Coulomb barrier is not surmounted, the nuclear interactions are also possible, but the distance between objects is much higher than $r_{S}$.

It is possible due to:

1) the tunneling effect, the probability of which is extremely small for large objects (bismuth-209 nuclei);

2) electromagnetic interaction between protons of the colliding nuclei during which nuclei are deformed. First of all, proton's radius is deformed, to be more precise, the proton size of the initially not-spherical bismuth209 nucleus. The probability of this event is high, since there are many protons in colliding nuclei and the range of electromagnetic interaction is infinite. In this case the distance of closest approach between the nuclei can be significantly higher than $r_{S}$. Just at this moment mutual Coulomb repulsion of positively charged ions (or nuclei) in the crystal lattice of macro-objects causes the inertial explosion of the metal (M. K. Marakhtanov, \& A. M. Marakhtanov, 2014). Before the explosion, the common electrons of crystal lattice have been lost, ions located in lattice sites can loose their electrons completely or partially and immediately the high number of nuclei can be deformed practically simultaneously. In addition to nuclear processes, $\beta$ transformations (atomic electron is caught by nucleus's proton) can occur with high probability. The high pressure caused by macro-objects high-velocity collision $(\sim 1 \mathrm{~km} / \mathrm{s})$ promotes this process. Thus, strong and weak nuclear processes can be initiated by electromagnetic processes.

\subsection{Shape of Atomic Nucleus: Variety and Discreteness}

Each energy state corresponds to a certain geometric shape of the nucleus. The energy levels are discrete and it means that the nuclear shapes are also "discrete". It is possible to number the nuclear shapes as well as the energy levels. Zero corresponds to the ground state (zero excitation energy), "1" corresponds to the first excited state (to the state with minimal excitation energy differed from zero), etc. The energy levels are fuzzy according to the Heisenberg uncertainty principle and they are overlapped under high excitation energies. Nuclei shapes are also determined within the Heisenberg uncertainty principle. The transition from one geometric shape of the nucleus to the other one is performed unevenly. Under high excitation energies in the region of overlapping levels, such transition is practically continuous. The smaller excitation energy corresponds to a smaller deformation of the shape with respect to the ground energy state. If the excitation energy increases, the nucleus's deformation also increases. Under low excitation energy (small deformation with respect to the ground state), for the nucleus it is sufficient to emit one or several gamma quanta for transiting to the ground state. If the excitation energy increases (the degree of deformation with respect to the ground state), first of all the probability for emitting an individual nucleon increases, after that the probability for emitting a group of nucleons (possibly in the form of the ${ }^{2} \mathrm{H}$ or ${ }^{3} \mathrm{H}$ fragment) rises, after that the possibility for emitting $\alpha$-particles of a heavier nuclear cluster increases, and finally, the heavy nucleus can fission. Nucleus changes its shape under transiting to another energy level.

\subsection{Deformation as a Factor of Nucleus Destabilization}

Any deformation of the atomic nucleus independently on the reasons, which can cause such deformation (nucleus transition from the ground state into excited energy state by its irradiation by hadrons, gamma quanta and by other particles, anomalously high temperature and pressure including caused by macro-objects collisions) changes spin and intrinsic quadrupole electric moment $Q$ of the nucleus, and can destroy the shell structure of the nucleus and changes the energy levels arrangement. As a result the nucleus stability decreases $\left(T_{1 / 2}\right)$. Nucleus deformation can 
disturb atom's electron shell (which has been already disturbed by macro-objects collision) protecting the nucleus against these impacts. For example, high-velocity collision of macro-objects containing depleted uranium deforms uranium-238 nuclei, decreases half-lifetime by orders (also with respect to spontaneous fission) and initiates nuclear reactions by decay products (by neutrons and gamma quanta) (Marakhtanov \& Okunev, 2016).

The reason of nucleus deformation is not important, it is important that the nucleus is in the deformed state with respect to the ground energy state. This deformed state can be associated with a specific excitation energy $E$ or with a certain energy level. As it is mentioned above the excitation can be stopped by particle emission: by gamma quantum, single nucleon and by nuclear and fission fragments. As a result the nucleus can decay. The probability of this or that decay way depends on energy $E$ and nucleus shape. Hereby, nuclei decay can be initiated by nuclei deformation caused by mechanical collisions of macro-objects containing these nuclei. Not a single nucleus decays. The process is of a group (collective) nature. Other things being equal (shape, macro-objects' composition) a certain decay way should be prevalent under given collision velocity of macro-objects. It is possible to accelerate a certain type of radioactive decay and to slow down other types of decay by choosing the collision velocity. It is possible to initiate extremely rare processes in nature: proton or two-proton radioactivity, cluster decay.

It is possible to see stable nuclei decay by colliding macro-objects under velocities insufficient for surmounting the mutual Coulomb repulsion of nuclei. Per se, this is a new class of physical processes: collective radioactive decays of atomic nuclei initiated by external mechanical impact (by temperature or pressure).

\subsection{Mechanisms of Decay}

It is possible to propose two mechanisms of atomic nuclei decay into fragments initiated by mechanical collision of macro-objects containing these nuclei. Let us examine cluster decay as an extremely rare process in nature.

\subsubsection{Light Cluster Formation from Quasi-Free Neutrons}

The nucleus of bismuth-209 is characterized by the magic number of neutrons $(\mathrm{N}=126)$, therefore, $\mathrm{Q}(\mathrm{N})=0$, and by not magic number of protons $(Z=83)$, hence $Q(Z) \neq 0$. The neutron matter in bismuth-209 nucleus "tends" to form a sphere and proton matter "tends" to deform this sphere. Due to nuclear (strong) forces of nucleons mutual attraction, the nucleus, which is even in the ground state of energy, is deformed, and its neutron and proton dimensions (radii) are different. First of all the electromagnetic interaction is observed at the distances between approaching nuclei $r>r_{s}$. (The range of the electromagnetic interaction is infinite). On contrast to protons, neutrons have no electric charge and their participation in electromagnetic interaction is lower. Therefore, nuclear matter containing protons is much more deformed. Usually, in the central part of the nucleus $\mathrm{N} \approx \mathrm{Z}$. The peripheral region of the nucleus is over-populated by neutrons. (In the nucleus of bismuth-209: $N>Z, N-Z=43$ ). Nuclei are deformed by mutual Coulomb repulsion of protons in neighboring nuclei under their approaching each other. The process is of a collective nature: many nuclei interacts each other at once.

Hereby, in the case of a mechanical collision of macro-objects, the proton radius (size) of the nucleus is deformed greatly due to electromagnetic interaction. As a result, a neutron "cloud" is formed in the deformed nucleus near the surface. This is a region (regions) consisting only of neutrons: $N / Z>>1$. Neutrons of this region are bonded each other and with the central part of the nucleus by nuclear attraction, which is lower than it is in the central part of the nucleus. As a result, the nucleus can emit these neutrons (let us call them quasi-free ones). Under high pressure caused by macro-objects collision with velocities of $\sim 1 \mathrm{~km} / \mathrm{s}$, remnants of electron cloud approach to the nucleus. If the deformation causes even greater deviation from the spherical shape of the bismuth-209 nucleus, the remaining atomic electrons approach much closer to nucleus's protons. Hereby, it is very probable that the atomic electron is captured by nucleus's proton.

At the time before quasi-free neutrons escape the nucleus, the neutron cloud is confined by mutual nuclear attraction of neutrons and the heavy fragment remained after bismuth-209. For light nucleus formation a part of neutrons of the cloud should be transformed into protons and this process is the most efficient in terms of energy. The process is implemented according to symmetry energy: the tendency to stabilize nuclei at $\mathrm{N}=\mathrm{Z}$. It is revealed that 10-12 neutrons evaporate under lead and bismuth induced fission (Shpolski, 1948; Goeckermann \& Periman, 1949). If the number of quasi-free neutrons, for example, is 11 , the transmutation of five neutrons into five protons $\left(5^{1} \mathrm{n} \rightarrow 5^{1} \mathrm{p}\right)$ corresponds to the boron-11 nucleus formation. The transmutation of six neutrons $\left(6{ }^{1} \mathrm{n} \rightarrow 6{ }^{1} \mathrm{p}\right)$ corresponds to carbon-11 nucleus formation (which immediately decays into boron-11). Boron-11 or carbon-11 nuclei are formed near the heavy fragment remained after bismuth-209. (Since several protons can be among quasifree neutrons, the number of such transitions should be lower than five). The heavy cluster (bismuth-198) remained after bismuth-209 nucleus decays into platinum-198 by means of weak interaction. Finally we have as follows: ${ }^{209} \mathrm{Bi} \rightarrow{ }^{198} \mathrm{Pt}+{ }^{11} \mathrm{~B}$, where the initial nucleus and products of its decay are stable. 
It is probable that the nucleus emits quasi-free neutrons. These neutrons can initiate the nuclear reactions.

Let us point out that in nuclear reactions of fragmentation usually the light clusters are stable and heavy ones are either radioactive since neutron is over-populated by neutrons relatively to optimal N/Z, or stable (Perfilov, Lozshkin, \& Shamov, 1960; Okunev, 2016). Hereby, decays occurring in other ways are also possible, but if the light stable cluster with optimal N/Z is formed, the heavy cluster is also characterized by N/Z close to optimal one. Due to this fact on contrast to the natural cluster radioactivity, it is more probable that light clusters with small mass number appear under mechanical collision of macro-objects. At the same time the approaching velocity of colliding macro-objects is the determining factor.

Under higher velocities of colliding macro-objects containing bismuth, we can expect the subsequent decay of the heavy clusters ${ }^{198} \mathrm{Pt}$ or ${ }^{198} \mathrm{Bi}$, which are excited, i.e. the decay process continues and cluster decay can transform into nuclear fission. In this case we should speak about induced fission of bismuth-209 initiated by mechanical collision of macro-objects. According to the nucleus drop model, the spontaneous fission of bismuth-209 is energetically favorable, but it is impeded by the energy barrier (Okunev, 2015).

\subsubsection{Nucleus Direct Decay into Clusters}

Another decay mechanism is analogous to the process of cluster radioactivity or nuclear reaction of highly asymmetric nuclear fission (fragmentation). Bismuth-209 nucleus deformation caused by mechanical collision of macro-objects is analogous to nucleus transition into excited state, for which ${ }^{209} \mathrm{Bi} \rightarrow{ }^{198} \mathrm{Pt}+{ }^{11} \mathrm{~B}$ is very probable. Such direct decay should take place under collision velocities lower than it is for ${ }^{209} \mathrm{Bi} \rightarrow{ }^{298} \mathrm{Bi}+11^{1} n$ with further transmutation $5^{1} \mathrm{n} \rightarrow 5^{1} \mathrm{p}$ and boron-11 formation. The mechanism of bismuth direct decay into clusters is same to the natural cluster radioactivity described by nucleus drop model. (The nucleus-drop of a pear-shape decays into two parts with very different masses.) In this case, the decay products are stable and in the system there are no free neutrons able to initiate nuclear reactions.

The mechanisms of fission, $\alpha$-decay, and decays of bismuth-209 into other clusters under mechanical collisions of macro-objects are the same.

\section{Experiments}

\subsection{Experimental Conditions. Materials}

Direct impact experiments for studying bismuth-209 transmutation under mechanical collisions between bismuth bullet (impactor) and a steel flat target with thickness ranging from 10 to $35 \mathrm{~mm}$ were carried out in Ion-plasma Center of Plasma Power Plants Department of Bauman Moscow State Technical University in 2013-2016 years. Preliminary results are presented in (Marakhtanov, 2016; Baranov, Baranova, Dukhopelnikov, \& Marakhtanov, 2015; Marakhtanov, Veldanov, Maksimov, \& Tarasov, 2009; Marakhtanov et al., 2013; Dukhopelnikov et al., 2010; Marakhtanov, 2009).

As a bullet material we use alloy of $97.89 \% \mathrm{Bi}+0.91 \% \mathrm{In}+0.74 \% \mathrm{Sb}+0.36 \% \mathrm{Fe}+0.11 \% \mathrm{Cr}+$ less than $0.16 \%$ $\mathrm{Pb}+0.04 \% \mathrm{Ni}$ (weight fractions are indicated). This alloy corresponds to the brand "Vi2" (Russian State Standard 10928-90) with pure bismuth content of $97-98 \%$.

We use steel AK 25 containing ferrum (about of 95\%) and doping elements Ni, Cr, C, Si, Mn, Mo, Cu, S, P as the material of the flat barrier with thickness of $10 \ldots 35 \mathrm{~mm}$ (Marakhtanov, Veldanov, Maksimov, \& Tarasov, 2009; Marakhtanov et al., 2013; Marakhtanov, 2016). The steel target is used for performing proper experiment. Steel contains mainly the most stable "average-mass" nuclei with maximal specific binding energy per nucleon.

Four experimental series with different velocities of bismuth bullets: series A (velocity of about $791 \mathrm{~m} / \mathrm{s})$, B (1224 $\mathrm{m} / \mathrm{s}), \mathrm{C}(1095 \mathrm{~m} / \mathrm{s}), \mathrm{D}(956 \mathrm{~m} / \mathrm{s})$ are of the great interest for investigating bismuth decay.

\subsection{Results}

Direct impact experiments initiate inertial bullet explosion, which knock out a steel plug from a steel barrier. Bullet explosion is accompanied by bright flash in the zone of its contact with the obstacle. The plugs knocked out of the steel barrier look like a cylinder, one base of which is a plane of contact with bullet's material. The lateral surface of the plugs is close to cylindrical one. In all series (A-D), we see cluster decay of stable bismuth isotope into two stable nuclides: ${ }^{209} \mathrm{Bi} \rightarrow{ }^{198} \mathrm{Pt}+{ }^{11} \mathrm{~B}$. Platinum and boron are present at the base of the knocked-out cylinder contacting with the bismuth bullet (Marakhtanov, 2016).

Experiments are characterized by repeatability. The process of cluster radioactivity is a mass phenomenon. $\alpha$ particles with energy of about $8 \mathrm{MeV}$ emitted by metallic dust formed directly at the site of interaction between bismuth bullet and steel (Marakhtanov, 2016; Baranov, Baranova, Dukhopelnikov, \& Marakhtanov, 2015; Marakhtanov, Veldanov, Maksimov, \& Tarasov, 2009; Marakhtanov et al., 2013; Dukhopelnikov et al., 2010; 
Marakhtanov, 2009) are recoded in B series. The most probable source of delayed high-energy $\alpha$-particles can be bismuth-198 nuclei formed according to process ${ }^{209} \mathrm{Bi} \rightarrow{ }^{198} \mathrm{Bi}+11^{1} \mathrm{n}$. This process is promoted by bullet's high velocity. In this case, we can speak about incomplete fission of bismuth-209 initiated by the external impact. Only the first stage of fission is implemented: ${ }^{209} \mathrm{Bi} \rightarrow{ }^{198} \mathrm{Bi}+11{ }^{1} \mathrm{n}$ accompanied by $\alpha$-decay, not by bismuth- 198 fission.

\subsection{Equipment for Identifying the Decay Products}

Four diagnostic groups and the following diagnostic devices are used for identifying the products caused by bismuth bullet interaction with the target:

1) XRF-1 is X-Rays Fluorescent spectrometer "Bruker S1 Titan" (produced by Bruker AXS Handled Inc., USA). The spectrometer detects platinum on the back surface of all plugs. But the mass share of platinum is overestimated since platinum and bismuth lines are close in the x-ray spectrum of condensate.

2) XRF-2 ("EXP-1; XRF Experimenter's Kit") is X-ray fluorescence spectrometer (produced by Amptek Inc., USA). It is called the $\mathrm{X}$-ray detecting system $X-123 S D D$. This system consists of silicon drift detector (SDD) and Mini-X USB controlled X-Ray Tube. With the help of these instruments it becomes possible to recognize platinum lines position in condensate spectrum for the energy ranging from 8 up to $10 \mathrm{keV}$. The system detects as follows: platinum is $0.089 \mathrm{mg}$ per $1 \mathrm{~cm}^{2}$ of condensate layer's surface.

3) LAES is Laser-Atomic Emission Spectrometer LAES MATRIX SPECTROMETER for analyzing emission lines of laser plasma spectrum (plasma is generated from a condensate material by laser beam energy). The experiment shows that in the condensate spectrum there are four platinum lines with wavelengths of 201.534; 204.953; 214.402 and $224.592 \mathrm{~nm}$.

Boron line $(206.571 \mathrm{~nm})$ and platinum line $(206.750 \mathrm{~nm})$, which has no bismuth lines in the nearest neighbor, are clearly seen. The steel target, from which the plugs are knocked out, includes ferrum $(216.175 \mathrm{~nm}$ and $206.367 \mathrm{~nm})$, cuprum $(206.604 \mathrm{~nm})$ and nickel $(206.835 \mathrm{~nm})$. The lines of these metals are sufficiently far from the boron line $(206.571 \mathrm{~nm})$ for distorting boron line formation. There are no boron and platinum in bullet and target composition, but their lines are clearly seen in the spectrum of products generated by their interaction.

4) SEM is Scanning Electron Microscope JSM-6060A (JEOL, Japan) with an energy dispersive attachment JED-2300 (JEOL, Japan) for recognizing boron in the condensate. SEM-diagnostic of plugs surface is performed only for confirming boron presence in condensate. Measurements are performed in ten points at the back surface of one plug. Each measurement shows boron presence with mass content ranging from $7.93 \%$ up to $11.20 \%$. Under examining another plug, eight points are probed. Boron presence with mass content ranging from $10.29 \%$ up to $15.76 \%$ is detected in seven points. It is necessary to point out that it is difficult to perform quantitative micro-analysis of light elements $(\mathrm{B}, \mathrm{C}, \mathrm{N}, \mathrm{O})$ since low-energy $\mathrm{X}$-rays emitted by the sample is absorbed strongly. Hereby, this instrument makes it possible to estimate boron presence in condensate only qualitatively.

The main disadvantage of elemental analysis of the inertial explosion condensate is as follows: the number of methods used for diagnostic is small, in particular, spectroscopy is not used.

\section{Discussion. Comparison of the Examined Physical Processes with Known Ones}

The presented mechanism of stimulated cluster decay of stable bismuth-209 nucleus caused by macro-objects collision is in good agreement with the results obtained in 1940s-1950s and related to nuclear reactions of fission and fragmentation of bismuth (see Shpolski, 1948; Goeckermann \& Periman, 1949; Perfilov, Lozshkin, \& Shamov, 1960; Serber, 1947; Fujimoto \& Yamaguchi, 1950; and references to these papers).

Cluster decay of nuclei initiated by macro-objects collision is similar to nuclear reactions of fragmentation, stimulated fission, and spontaneous cluster decay. At the same time, there are differences between these processes.

Table 1 depicts the main similarities and differences of the nuclear fragmentation reaction (cluster emission) and cluster decay initiated by mechanical collision of macro-objects.

Similarities are as follows. On contrast to the fission process, for which two fragments formation is the most probable, the process of cluster emission is characterized by multiplicity. The cross section (probability) of clusters formation increases sharply, if the energy of incident particle increases. As a rule the light clusters are in the ground energy state. 
Table 1. Differences between fragmentation reaction and cluster decay initiated by mechanical collision of macroobjects

\begin{tabular}{|c|c|c|}
\hline \multicolumn{3}{|c|}{ Observable regularities } \\
\hline No & $\begin{array}{l}\text { The nuclear fragmentation reaction (see Perfilov, } \\
\text { Lozshkin, \& Shamov, } 1960 \text { and references to this } \\
\text { work) }\end{array}$ & Cluster decay initiated by macro-objects collision \\
\hline 1 & $\begin{array}{l}\text { If light cluster's mass decrease, its yield } \\
\text { increases }\end{array}$ & $\begin{array}{l}\text { Cluster's yield depends on the velocity of macro- } \\
\text { objects collision }\end{array}$ \\
\hline 2 & $\begin{array}{l}\text { As a rule the light clusters are stable, and the } \\
\text { residual nucleus is either over-populated by } \\
\text { neutrons (radioactive) or stable }\end{array}$ & $\begin{array}{c}\text { Usually if bismuth-209 decays, both clusters are } \\
\text { stable. If heavier nuclei decay, the residual } \\
\text { nucleus is over-populated by neutrons }\end{array}$ \\
\hline 3 & $\begin{array}{l}\text { The cross section (probability) of clusters' } \\
\text { formation increases slowly, if the mass number } \\
\text { of the target nucleus increases }\end{array}$ & $\begin{array}{l}\text { The cross section (probability) of clusters' } \\
\text { formation increases sharply under lead stable } \\
\text { isotopes transformation into to bismuth }\end{array}$ \\
\hline
\end{tabular}

Table 2 makes it possible to compare spontaneous cluster decays and decays initiated by mechanical collision of macro-objects. The similarity is as follows: both types of decay (spontaneous and initiated by mechanical collision of macro-objects) are intermediate between $\alpha$-decay and fission.

Table 2. Differences between spontaneous cluster decay and cluster decay initiated by mechanical collision of macro-objects

\begin{tabular}{|c|c|c|}
\hline \multicolumn{3}{|c|}{ Observable regularities } \\
\hline No & $\begin{array}{c}\text { Spontaneous cluster decay of heavy nuclei } \\
\text { (Sandulescu, Poenaru, \& Greiner, 1980; Rose \& } \\
\text { Jones, 1984; Alexandrov, Belyatsky, \& Glukhov, } \\
\text { 1984; Baum, Knox, \& Miller, 2002; Bonetti \& } \\
\text { Guglielmetti, 2007; Guglielmetti, 2008) }\end{array}$ & Cluster decay initiated by macro-objects collision \\
\hline 1 & $\begin{array}{l}\text { Emission of clusters with mass number } \mathrm{A}= \\
\qquad 12 \ldots 34\end{array}$ & $\begin{array}{l}\text { Emission of clusters with any mass number }(\mathrm{A}= \\
\qquad 1 \ldots 208)\end{array}$ \\
\hline 2 & $\begin{array}{l}\text { Emission of certain clusters }\left({ }^{12} \mathrm{C},{ }^{14} \mathrm{C},{ }^{20} \mathrm{O},{ }^{23} \mathrm{~F} \text {, }\right. \\
\left.{ }^{24} \mathrm{Ne},{ }^{25} \mathrm{Ne},{ }^{26} \mathrm{Ne},{ }^{28} \mathrm{Mg},{ }^{29} \mathrm{Mg},{ }^{30} \mathrm{Mg},{ }^{32} \mathrm{Si} \text { and }{ }^{34} \mathrm{Si}\right)\end{array}$ & $\begin{array}{c}\text { The emission of any clusters, but emission of } \\
\text { light clusters with small mass number is the most } \\
\text { probable }\end{array}$ \\
\hline 3 & $\begin{array}{l}\text { In the majority of cases, the emitted light clusters } \\
\text { are radioactive }\end{array}$ & $\begin{array}{c}\text { Under }{ }^{209} \mathrm{Bi} \rightarrow{ }^{198} \mathrm{Pt}+{ }^{11} \mathrm{~B} \text {, the emitted clusters } \\
\text { (light and heavy) are stable }\end{array}$ \\
\hline 4 & $\begin{array}{l}\text { The probability of the process is extremely small: } \\
\text { much less than other types of decay }\end{array}$ & $\begin{array}{l}\text { Process probability depends on the velocity of } \\
\text { macro-objects collision and under certain } \\
\text { velocities it can be close to } 100 \%\end{array}$ \\
\hline
\end{tabular}

\section{Conclusion}

The shape of the atomic nucleus can be deformed, if the nuclei approach each other to a distance significantly higher than the range of nuclear forces, i.e. it is not necessary to surmount the Coulomb barrier. It is important for relatively low collision velocities $(\sim 1 \mathrm{~km} / \mathrm{s})$. Nucleus deformation by the external mechanical action (impact) to macro-object corresponds to the shape of excited nucleus. The "excitation" (i.e. the shape deformation with respect to the ground state) is stopped by nucleus decay similar to nucleon (or a group of nucleons) emission (including fission isomerism of heavy nuclei) from highly excited state. 
The processes of cluster decay initiated by the mechanical collision of macro-objects are differed from the known reactions of fragmentation and cluster radioactivity. Bismuth is not the only one material for which one can observe the accelerated decay of atomic nuclei. Similar phenomenon is typical for materials containing nuclei, which is much heavier than ferrum and nickel, and especially for heavy long-lived nuclei. For example, macro-objects collision accelerates uranium-238 decay by many times (including spontaneous fission) (Marakhtanov \& Okunev, 2016).

Since the range of electromagnetic interaction is infinite, of nuclear interactions is not higher than $10 \mathrm{fm}$, of weak interaction is about of $0.2 \mathrm{fm}$, it is possible to assume that if macro-objects with crystalline structure approach each other with high velocity (sufficient for inertial explosion, but insufficient for surmounting the Coulomb barrier under nuclei approach) the nuclei in the bullet and (or) in the target can interacts only electromagnetically. The gravitation interaction is not able to change physical picture due to its extremely low intensity.

The experiments and theoretical analysis makes it possible to assume that there are a whole class of new physical processes: collective decays of atomic nuclei (including stable ones) initiated by external mechanical impact (by temperature or pressure) when the nuclei approach each other at the distance higher than the radius of nuclear interaction. In other words it is possible to accelerate decay and atomic nuclei transformation without using charge particles accelerators, nuclear reactors and ionizing radiation, i.e. by hammer, pressure or temperature impacts.

The obtained results can be used for developing the new environmentally friendly plants for generating small quantity of energy, for synthesizing rare elements and for researching extremely rare natural processes: cluster decays.

"Collective radioactive decays of atomic nuclei initiated by external mechanical action": is it the science fiction or a new class of physical processes? Only the mass spectroscopy is able to answer this question.

\section{References}

Alexandrov, D. V., Belyatsky, A. F., \& Glukhov, Yu. A. (1984). JETP Lett., 40(4), 152-154.

Audi, G., Wapstra, A. H., \& Thibault, C. (2003). The AME2003 atomic mass evaluation:(II). Tables, graphs and references. Nuclear physics A, 729(1), 337-676.

Baranov, D. S., Baranova, O. D., Dukhopelnikov, D. V., \& Marakhtanov, M. K. (2015). In Proc. VIII Conf. "NonReversible Processes in Nature and Technics", Moscow, 2015 (vol. 1, pp. 248-252).

Baum, E. M., Knox, H. D., \& Miller, T. R. (2002). Nuclides and Isotopes: Chart of the Nuclides (16th ed.). Knolls Atomic Power Laboratory (Lockheed Martin).

Bhatki, K. S. (1977). Radiochemistry of bismuth (No. NAS-NS-3061). Tata Inst. of Fundamental Research, Bombay (India).

Bonetti, R., \& Guglielmetti, A. (2007). Cluster radioactivity: an overview after twenty years. Romanian reports in Physics, 59(2), 301.

Dukhopelnikov, D. V., Kalashnikov, N. P., Marakhtanov, A. M., Marakhtanov, M. K., \& Olchak, A. S. (2010). Nucl. Phys. Eng., 4, 339.

Fujimoto, Y., \& Yamaguchi, Y. (1950). Note on Very Large Cosmic-Ray Stars. Progress of Theoretical Physics, $5,76-81$.

Goeckermann, R. H., \& Periman, I. (1949). Phys. Rev., 78, 629.

Guglielmetti, A., Faccio, D., Bonetti, R., Shishkin, S. V., Tretyakova, S. P., Dmitriev, S. V., ... \& van der Walt, T. N. (2008). Carbon radioactivity of $223 \mathrm{Ac}$ and a search for nitrogen emission. In Journal of Physics: Conference Series (Vol. 111, No. 1, p. 012050). IOP Publishing.

Marakhtanov, M. K. (2009). Izv. RAN. Energ., 1, 79.

Marakhtanov, M. K. (2016). Possible nuclear decay of bismuth induced by a mechanical impact. Russian Metallurgy (Metally), 2016(9), 884-888.

Marakhtanov, M. K., \& Marakhtanov, A. M. (2014). Quantum Macro-Electronics: Events of Macro-World, Explicable by Lows of Quantum Mechanics. Experience and Theory, Moscow: KRASAND. (in Russian)

Marakhtanov, M. K., \& Okunev, V. S. (2016). Influence of mechanical collisions of macroobjects on the nuclearphysical properties of constituent and heavy nuclides. Bulletin of the Moscow State Technical University. NE Bauman. Series "Natural Sciences", 1(64). 
Marakhtanov, M. K., Veldanov, V. A., Dukhopelnikov, D. V., Karneychik, A. S., \& Maksimov, M. A. (2013). Inz. Zh.: Nauka Innov., 9, 1.

Marakhtanov, M. K., Veldanov, V. A., Maksimov, M. A., \& Tarasov, M. A. (2009). Izv RARAN., 1(59), 43.

Okunev, V. S. (2015). The basis of applied nuclear physics and introduction into physics of nuclear reactors, Ser. "Physics in Technical University", Bauman Moscow State Technical University. (in Russian)

Okunev, V. S. (2016). Some Paradoxes and Laws of Low-Energy Nuclear Physics. Bauman Moscow State Technical University. (in Russian)

Perfilov, N. A., Lozshkin, O. V., \& Shamov, V. N. (1960). Usp. Phys. Nauk, 70(1), 3.

Rose, H. J., \& Jones, G. A. (1984). Nature, 309, 245-247.

Sandulescu, A., Poenaru, D. N., \& Greiner, W. (1980). Sov. J. Part. Nucl., 11, 528-541..

Serber, R. (1947). Nuclear reactions at high energies. Physical Review, 72(11), 1114.

Shpolski, E. (198). Usp. Phys. Nauk, 34(3), 440.

\section{Copyrights}

Copyright for this article is retained by the author(s), with first publication rights granted to the journal.

This is an open-access article distributed under the terms and conditions of the Creative Commons Attribution license (http://creativecommons.org/licenses/by/4.0/). 